\title{
AUTOMATIC ESTIMATION OF SIZE PARAMETERS USING VERIFIED COMPUTERIZED STEREOANALYSIS
}

\author{
PETER R MOUTON ${ }^{1,3}$, JOEl DURGAVICH$^{2}$ AND DONALd K INGRAM ${ }^{3}$ \\ ${ }^{1}$ Stereology Resource Center, 104 Ringneck Court, Chester MD 21619, src@disector.com; ${ }^{2}$ Systems Planning \\ and Analysis, Inc., Alexandria, VA, 22311, stereology@spa.com; ${ }^{3}$ Laboratory of Experimental Gerontology, \\ National Institute on Aging, National Institutes of Health, 5600 Nathan Shock Drive, Baltimore, MD 21224, \\ ingramd@grc.nia.nih.gov \\ e-mail: peter@disector.com \\ (Accepted Jannuary 28, 2004)
}

\begin{abstract}
State-of-the-art computerized stereology systems combine high-resolution video microscopy and hardwaresoftware integration with stereological methods to assist users in quantifying multidimensional parameters of importance to biomedical research, including volume, surface area, length, number, their variation and spatial distribution. The requirement for constant interactions between a trained, non-expert user and the targeted features of interest currently limits the throughput efficiency of these systems. To address this issue we developed a novel approach for automatic stereological analysis of 2-D images, Verified Computerized Stereoanalysis (VCS). The VCS approach minimizes the need for user interactions with high contrast [high signal-to-noise ratio (S:N)] biological objects of interest. Performance testing of the VCS approach confirmed dramatic increases in the efficiency of total object volume (size) estimation, without a loss of accuracy or precision compared to conventional computerized stereology. The broad application of high efficiency VCS to high-contrast biological objects on tissue sections could reduce labor costs, enhance hypothesis testing, and accelerate the progress of biomedical research focused on improvements in health and the management of disease.
\end{abstract}

Key words: image analysis, stereoanalysis, unbiased stereology, volume fraction.

\section{INTRODUCTION}

The sustained efforts of stereologists for the past four decades developed design-based techniques for quantification of arbitrary-shaped biological features in tissue sections. These methods included unbiased sampling schemes in combination with geometric probes to quantify first- and second-order stereological parameters of interest to biomedical research, including feature number, length, surface area, volume, their variation and spatial distribution. The mathematical foundations of these theoretically unbiased approaches draw heavily on stochastic geometry and probability theory, in contrast to earlier so-called assumptionand model-based approaches that apply poorly to arbitrary-shaped biological features (Gundersen et al., 1988a,b; Mouton, 2002; Hilliard and Lawson, 2003). In the middle 1990s the emerging consensus by the biomedical research community began to openly favor the design-based stereological methods, where possible, for the morphological analysis of tissue sections:
"Stereologically based unbiased estimates are always preferable for establishing absolute counts or densities of structures in tissue sections. We expect that any papers that use simple profile counts, or assumption-based correction factors, will produce adequate justification for these methods. Referees are urged to... insist on unbiased counts when this justification is not adequate" (Saper, 1996).

This preference for stereological approaches continued into the new decade, stimulating demand for high-throughput computerized stereological systems. The development of computer stereology software linked to acquisition of real-time and stored microscopic images dramatically improved the throughput efficiency of data collection using design-based stereology (Fig. 1). The recent availability of low-priced, high quality hardware for these systems, including high-resolution microscopy and video card technology, motorized XY-Z stage control, and PC- or Macintosh-based computing power, further improved the accessibility of these systems by rank-and-file biomedical scientists. 


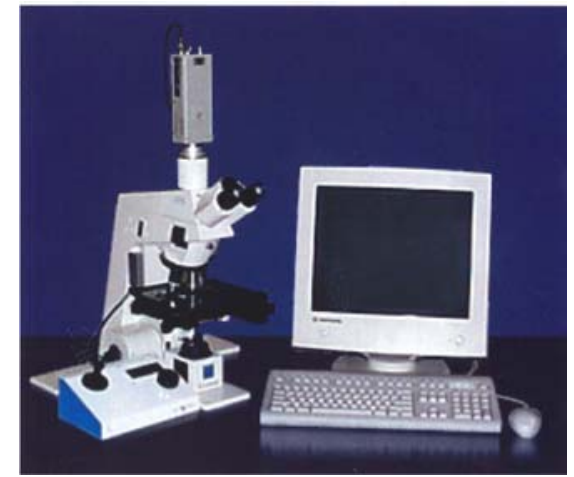

Fig. 1. A computerized stereology system (Stereologer, Systems Planning and Analysis, Inc., Alexandria, VA).

In recent years the user of computerized stereology have focused critical attention on the rate-limiting step in the efficiency of systems: The requirement for tedious, costly, and labor-intensive identification of targeted features by non-expert users. Overcoming this limitation, without introducing non-verifiable assumptions or models, would enhance the throughput efficiency of computerized stereology while reducing labor costs and accelerating the progress of biomedical research.

To address this issue we developed a novel hybrid program, Verified Computerized Stereoanalysis (VCS), which involves a two-step combination of image analysis and unbiased stereology: auto-detection of high contrast images of biological interest based on high signal-to-noise (S:N) ratio, followed by autoanalysis of images using state-of-the-art stereological principles. Operationally, the VCS approach involves the acquisition of an internal range of target color pixels associated with the high contrast feature of interest while the user carries out conventional procedures of computerized data collection on the first section of the initial case in a study. Performance testing of the VCS program confirmed dramatic improvements in efficiency of volume (size) parameter estimation compared to conventional computerized stereology approaches.

\section{MATERIALS AND METHODS}

\section{HARDWARE-SOFTWARE COMPONENTS}

For testing purposes, algorithms for automatic VCS were developed and incorporated into a PC- and Mac-compatible computerized stereology system (Stereologer, Systems Planning and Analysis, Inc., Alexandria, VA). The integrated hardware-software components of the system includes the following:
- Light microscopy images captured and displayed on the computer monitor using a high-end video card and digital video camera.

- Accurate stage movement in three dimensions via motorized $\mathrm{X}-\mathrm{Y}-\mathrm{Z}$ stage and controller.

- Software integration for simultaneous management of devices and implementation of stereology and image analysis algorithms.

\section{VCS Menu}

The program provides access to the VCS routines using three commands:

1. Display the Settings dialog box.

2. Execute Semi-Automatic Mode to collect data for current probe at current $x-y$ location.

3. Execute Automatic Mode to collect data for current probe at all locations on the current section.

\section{SETTINGS DIALOG BOX}

Using the Setting dialog box the user trains the software to recognize the specific color pixel spectrum for the target images. The dialog box is split into three sections: color control, feature size, and pixel resolution. The Color Control dialog (Fig. 2) selects the desired probe-feature intersections based on the relative contrasts between red-green-blue pixel components for the various features in the sampling frame.

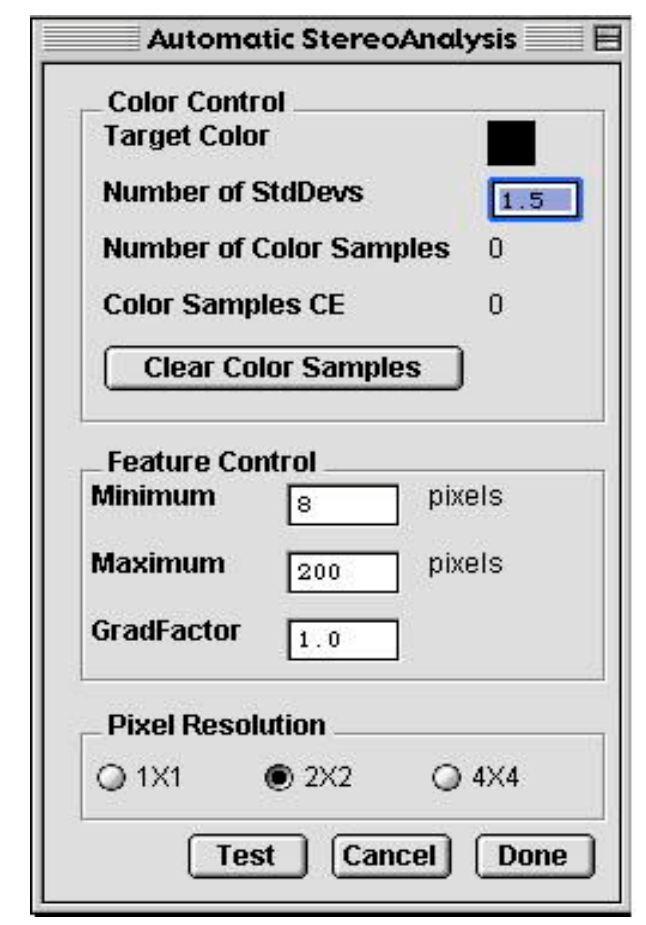

Fig. 2. Dialog box for VCS Settings. 
The range of target pixels is a function of the average of sampled colors and the proximity to the target color, as determined by user-selected standard deviations (SD) from the selected color samples to the target color. These studies verified that for high contrast features, optimal feature identification to $100 \%$ accuracy was achieved in the range of 1.5 to 2.0 SD from the central tendency of targeted pixels. The Clear Color Samples button clears the collected color samples to allow retraining the program. The Feature Size Control minimizes unwanted outliers from inclusion as a targeted intersection. Pixel Resolution Control averages several pixels together as a single pixel. Merging pixels regulates the speed of the data processing since fewer pixel elements require less time, from about 0.5 seconds for $4 \times 4$, to about 1.5 seconds for the $1 \times 1$ setting. The majority of these studies were carried out using the $2 \times 2$ setting in $1.0 \mathrm{sec}$, which achieved an optimal balance between speed and performance for high contrast features.

\section{VCS DATA COLLECTION LOGIC}

The VCS program follows the following logic:

1. Capture the image within the sampling frame into memory.

2. Fill an internal array with the RGB (red, green, blue) color information for the captured image.

3. Sharpen the color image by removing all colors distant from the target color. The color distance is calculated at each pixel location by a simple Pythagorean rule, Color Distance $=\left(\left(\mathrm{R}_{\mathrm{p}}-\mathrm{R}_{\mathrm{t}}\right)^{2}+\right.$ $\left.\left(G_{p}-G_{t}\right)^{2}+\left(B_{p}-B_{t}\right)^{2}\right)^{(1 / 2)}$, where $p$ subscript values are the RGB values at a pixel location and the $t$ subscript values are the RGB values of the target color. The corresponding distance is then compared to the threshold defined by the user. Pixel locations which produce a color distance less than or equal to the threshold are retained, whereas pixel locations which produce a color distance greater than the threshold are cleared.

4. Fill an array with the gradient at each pixel location by examining the nearest neighbors in $\mathrm{x}$ and y, i.e., Sobel Edge Detection.

5. Locate the features of interest by examining the gradient array.

6. Mark intersections between the probe and the identified features of interest.

As an illustration, consider an array filled with values from $\mathrm{C} 1$ to $\mathrm{C} 10$ representing ten potentially different RGB values (Fig. 3).

\begin{tabular}{|c|c|c|c|c|c|c|c|c|c|c|c|c|c|c|c|}
\hline 3 & 3 & 10 & 3 & 3 & 6 & 10 & 6 & 4 & 3 & 3 & 8 & 10 & 10 & 8 & 6 \\
\hline 5 & 1 & 7 & 6 & 4 & 10 & 1 & 8 & 10 & 8 & 10 & 7 & 1 & 10 & 6 & 10 \\
\hline 9 & 1 & 2 & 2 & 2 & 2 & 6 & 6 & 5 & 8 & 5 & 9 & 6 & 5 & 9 & 4 \\
\hline 9 & 10 & 2 & 2 & 2 & 2 & 1 & 8 & 5 & 4 & 6 & 8 & 7 & 4 & 5 & 5 \\
\hline 7 & 4 & 2 & 2 & 2 & 2 & 6 & 7 & 1 & 6 & 8 & 7 & 9 & 5 & 1 & 10 \\
\hline 7 & 7 & 6 & 2 & 2 & 2 & 8 & 7 & 5 & 1 & 2 & 2 & 2 & 9 & 3 & 8 \\
\hline 6 & 4 & 5 & 1 & 1 & 6 & 9 & 10 & 9 & 10 & 2 & 2 & 2 & 6 & 4 & 7 \\
\hline 10 & 6 & 4 & 10 & 4 & 1 & 5 & 7 & 7 & 9 & 2 & 2 & 2 & 3 & 10 & 9 \\
\hline 1 & 3 & 3 & 7 & 1 & 4 & 10 & 5 & 1 & 1 & 2 & 2 & 2 & 8 & 10 & 8 \\
\hline 5 & 5 & 5 & 8 & 4 & 4 & 4 & 4 & 7 & 1 & 5 & 2 & 2 & 4 & 1 & 4 \\
\hline 7 & 3 & 4 & 3 & 1 & 2 & 2 & 3 & 3 & 7 & 1 & 8 & 8 & 9 & 3 & 10 \\
\hline 8 & 1 & 4 & 1 & 2 & 2 & 2 & 2 & 8 & 10 & 6 & 10 & 1 & 1 & 9 & 3 \\
\hline 5 & 4 & 1 & 7 & 2 & 2 & 2 & 2 & 6 & 1 & 1 & 9 & 6 & 10 & 4 & 5 \\
\hline 5 & 3 & 5 & 4 & 2 & 2 & 2 & 2 & 4 & 6 & 5 & 1 & 6 & 1 & 1 & 4 \\
\hline 8 & 9 & 3 & 10 & 1 & 4 & 6 & 9 & 5 & 4 & 8 & 8 & 5 & 6 & 9 & 9 \\
\hline 10 & 1 & 6 & 10 & 10 & 5 & 1 & 7 & 6 & 6 & 6 & 7 & 8 & 5 & 1 & 7 \\
\hline
\end{tabular}

Fig. 3. An internal array filled values ranging from $C 1$ to $C 10$ to represent potential $R G B$ values.

We will now specify that our target color value is the RGB value corresponding to $\mathrm{C} 2$ and that we require an exact match i.e. threshold equal to 0 . Since only array locations with $\mathrm{C} 2$ will produce a color distance of zero, only those locations are kept, the rest are zeroed. Finally, examination of gradients greater than zero generates an outline of the features (Fig. 4). This approach allows for the specific identification of the boundaries of individual target features.

\section{SEMI- AUTOMATIC AND AUTOMATIC MODES}

In order to ensure that the targeted color spectrum avoids automatic type 1 and type 2 statistical errors, i.e., mistaken "yes" and "no" selections, respectively, the user trains the software to autodetect and autoanalyze target features of interest at systematicrandom locations on the current section. In SemiAutomatic mode, the user approves the automatic selections for the current probe at the current $x-y$ location, prior to motorized movement to the next $x-y$ location. When the S:N ratio of the targeted feature is sufficient to support fully automatic data collection, as evidenced by $100 \%$ agreement between manual and automatic counting, the user switches to full Automatic Mode by selecting Collect Images from the VCS drop down menu. The software automatically drives the motorized stage to collect and analyze data for current probe at all locations on the current section. If additional sections from the same reference space are contained on other slides, the user must switch slides then continue with the above steps until all sections have been analyzed for the current case. 


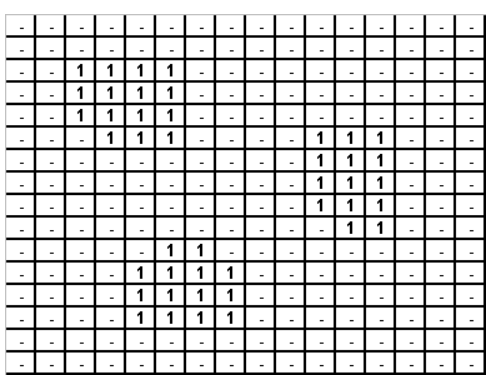

a)

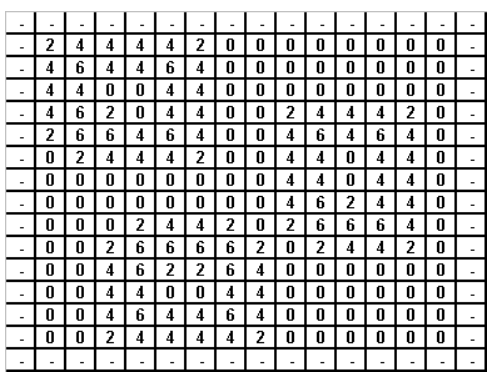

b)

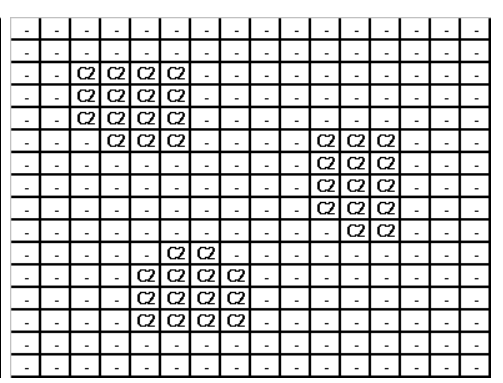

c)

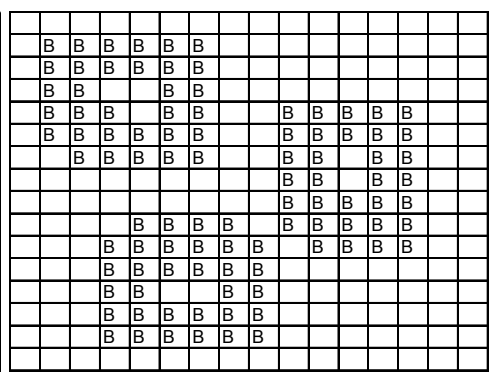

d)

Fig. 4. Color gradients smooth the feature detection process.

\section{VERIFICATION STUDIES}

Verification studies focused on the analysis of sections immunostained for bromodeoxyuridine (BrDU) from $\mathrm{n}=3$ mice. A total of 7-8 BrDU-stained sections were sampled in a systematic-random manner from the hippocampus of each mouse. Sections were cut on a freezing microtome at an instrument setting of 50 $\mu \mathrm{M}$. Immunostaining for BrDU followed the protocol detailed by Lee et al., (2003). This immunologicbased stain provides a high contrast stain of the nuclei of stem cells undergoing cell division, as shown in Fig. 5.

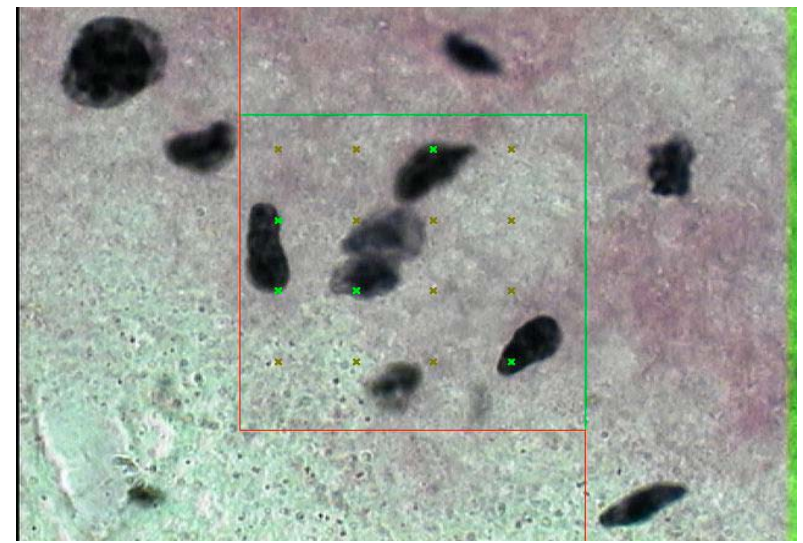

Fig. 5. A BrDU-stained cells showing probe-feature intersections ("+") using a point grid placed at random over the image. Note that the uppermost left point on the "+" must hit the feature for an intersection to be counted for either manual or VCS methods.
After complete tissue processing the sections had an average thickness of $19.5 \mu \mathrm{M}$ measured orthogonal to the tissue section by focusing on the top and bottom of the section using a $100 \times$ (N.A. 1.4) oil immersion objective.

The purpose of these studies was to assess the efficiency, accuracy, and precision of estimates using the gold-standard (conventional computerized stereology) compared to automatic and semi-automatic VCS for three size parameters: volume fraction $\left(\mathrm{V}_{\mathrm{obj}} / \mathrm{V}_{\text {ref }}\right)$, total volume of the reference space $\left(\mathrm{V}_{\text {ref }}\right)$, and total object volume, $\mathrm{V}_{\text {obj, }}$, as described below.

- $\mathrm{P}_{\text {object }} / \mathrm{P}_{\text {ref }}=\mathrm{A}_{\text {object }} / \mathrm{A}_{\text {ref }}=\mathrm{V}_{\text {object }} / \mathrm{V}_{\text {ref. }}$ According to the Delesse principle (Delesse, 1847), the ratio of object profile area $\left(\sum \mathrm{P}_{\text {object }} \cdot\right.$ area per point $)$ to area of reference area sampled $\left(\left(\sum \mathrm{P}_{\text {ref }} \cdot\right.\right.$ area per point) provides an unbiased estimate of the object volume fraction, $\mathrm{V}_{\text {object }} / \mathrm{V}_{\text {ref }}$ (Fig. 5). On random sections through high contrast of interest, the random intersection between a sum of points $\left(\sum \mathrm{P}\right)$ and 2-D profiles of targeted features is proportional to the volume of the feature in 3-D (Cavalieri, 1635; Glagolev, 1933; Gundersen et al., 1988 a,b; Mouton, 2002; Perry et al., 2004).

- Estimation of total reference volume, $\mathrm{V}_{\text {ref }}$, obtained by integration of area measurements on planes (physical or optical) a known distance apart, according to the Cavalieri principle $\left(\mathrm{V}_{\text {ref }}=\right.$ $\sum \mathrm{A}_{\text {section }}{ }^{\bullet}$ average distance between planes), as shown by Gundersen and Jensen (1987; for biological examples, see Mouton et al., 1998). 
- Estimation of total object volume, $\mathrm{V}_{\text {obj }}$, where $\mathrm{V}_{\text {obj }}=\left(\mathrm{V}_{\text {obj }} / \mathrm{V}_{\text {ref }}\right) \cdot \mathrm{V}_{\text {ref }}$, for thin or thick sections containing anatomically well-defined reference spaces that are $100 \%$ available for sampling. This includes reference spaces that can be systematically sampled in entirety, such as the majority of samples from mice, rats, non-human primates, and human tissue at autopsy. One example where this method would not be useful is when an unknown fraction of the reference space is available for sampling, i.e., tumor biopsies. In this case the expected volume would vary with the unknown volume of the tumor in situ, rather than the volume of the biopsy available for sampling.

\section{RESULTS}

Data are presented for timed estimations of volume fraction $\left(\mathrm{V}_{\mathrm{V}}\right)$ by three methods: manual point counting; VCS in semi-automatic mode (semiVCS); and, fully automatic VCS (autoVCS). Table 1 shows the time required for analysis by section using each of the three approaches applied to animal \#1.

As shown in Table 1 , a reliable $\mathrm{V}_{\mathrm{V}}$ estimate by manual point counting in animal \#1 required 141 minutes for analysis of $\mathrm{n}=8$ sections, including the actual time for analysis (excluding Study Set-up and Case Initialization), as outlined below.

1. Load slide onto motorized stage.

2. Outline the reference space at low power $(4 \times)$; switch to high power $(63 \times)$

3. Locate the top and bottom of the section.
4. Focus to the approximate mid-point of the section and manually select (click) each probe-feature intersection between an area point probe $(+)$ and the feature (BrDU-immunostained nucleus)

5. Select Next to move to the next $x-y$ position via motorized stage movement in $x-y$ axes.

6. Repeat steps 2-4 above for all frames spaced in a systematic-random manner across 8 sections.

For semiVCS, the user analyzed the same sections and followed the above steps 1-3 and 5-6. At step 4, the user selected Collect Data For Current Probe at each sampling frame. The program automatically selected points hitting BrDU-positive nuclei, with a total time of 90 min for analysis of $n=8$ sections for animal \#1. For autoVCS, the user followed the same steps 1 and 2 above, selected Clear Color Samples, then retrained the program to recognize BrDU-stained profiles. Once $100 \%$ agreement was achieved between automatic and manual data collection, the user selected Collect Data For Current Section. The same comparison was repeated for two additional animals. Table 2 shows the results for the analysis of BrDU-positive cells in animal \#2 for manual, semiVCS, and autoVCS methods.

Table 2 illustrates the increase in throughput efficiency for autoVCS from animals 1 to 2, due to the carry-over effect of training in the initial case to all subsequent cases in the same study. Table 3 compares results of throughput for animal 1-3. A total of $27 \mathrm{~min}$ was required to train the program to collect $\mathrm{V}_{\mathrm{V}}$ data for animal \#1. Carryover of training reduced the time to collect data for animal \#2 to $14 \mathrm{~min}$, reduction. Analysis of $\mathrm{V}_{\mathrm{V}}$ in animal \#3 only required $12 \mathrm{~min}$; again, because the training effect carried over from animal \#1 (Table 3).

Table 1. Data collection times by section for $V_{V}$ estimation of BRDU-immunostained cells in animal \#1.

\begin{tabular}{lccccccccc}
\hline Section\# & 1 & 2 & 3 & 4 & 5 & 6 & 7 & 8 & Total (min) \\
\hline Manual & 14 & 20 & 23 & 15 & 28 & 17 & 15 & 9 & 141 \\
SemiVCS & 12 & 15 & 15 & 10 & 14 & 12 & 8 & 4 & 90 \\
AutoVCS & 10 & 3 & 4 & 2 & 3 & 2 & 2 & 1 & 27 \\
\hline
\end{tabular}

Table 2. Data collection times by section for $V_{V}$ estimation of BrDU-immunostained cells in animal \#2.

\begin{tabular}{lccccccccc}
\hline Section\# & 1 & 2 & 3 & 4 & 5 & 6 & 7 & 8 & Total (min) \\
\hline Manual & 15 & 15 & 25 & 27 & 24 & 20 & 18 & 10 & 154 \\
SemiVCS & 9 & 10 & 14 & 15 & 10 & 11 & 9 & 3 & 81 \\
AutoVCS & 2 & 1 & 2 & 3 & 2 & 2 & 1 & 1 & 14 \\
\hline
\end{tabular}


The throughput analysis in Table 3 indicates that the rate of tissue analysis for autoVCS approximately doubled from about one-third of a section per minute on animal \#1, to a stable throughput of two-thirds of each section per minute for animals \#2 and \#3, as evidenced by results from efficiency testing across animals. Table 4 compares the analysis of $V_{V}$ for $n=3$ mice using manual point counting, semiVCS, and autoVCS approaches on the basis of precision (CE), as calculated using the quadratic approximation revised by Gundersen et al., (1999); and, efficiency $\left[(\mathrm{CE} /\right.$ time $\left.) \cdot 10^{3}\right]$.

These data confirm the efficiency of semiVCS and autoVCS compared to manual data collection. Furthermore, the results for autoVCS confirm that throughput increases as more cases are analyzed in the same study. Starting with the second section in animal \#1, VCS achieved 100\% agreement with manual point counting, allowing the user to analyze the remaining sections in the most efficient, fully automatic mode. For animals \#2 and \#3, the user verified $100 \%$ agreement on the first section, then carried out the analysis of remaining sections for each case in fully automatic mode. The final data for verification of total $\mathrm{V}_{\mathrm{V}}$ for BrDU-positive cells in $\mathrm{n}=3$ mouse hippocampi are shown in Table 5.

Table 5 shows the lack of differences for $\mathrm{V}_{\mathrm{V}}$ estimation of BrDU-positive cells for the three different approaches. From these estimates of $\mathrm{V}_{\mathrm{V}}$ the program automatically calculates total object volume, Total $\mathrm{V}_{\text {obj }}$, as the product of: $\mathrm{V}_{\mathrm{V}}\left(\mathrm{V}_{\text {object }} / \mathrm{V}_{\text {ref }}\right)$; and, the automatic estimate of total reference volume (Total $\mathrm{V}_{\text {ref }}$ ) (data not shown). The small amount of observed variation in mean $\mathrm{V}_{\text {object }} / \mathrm{V}_{\text {ref }}$ across different methods occurs from random sampling error (i.e., true variation between adjacent sampled regions).

Statistical studies with analysis of variance revealed significant differences in efficiency across the three methods $[\mathrm{F}(1,7)=23.22, \mathrm{p}<0.002)]$. Posthoc testing showed an eight-fold increase in efficiency for autoVCS compared to manual methods $(F(1,5)=$ $20.64, \mathrm{p}<0.01)]$; and, a significant doubling (2-fold) in efficiency for semiVCS compared to manual methods $(\mathrm{F}(1,5)=75.54, \mathrm{p}<0.001)]$. Comparable values for coefficient of error (CE) (Tables 4,5), a measure of within-sample precision, across the three different methods reflect the equivalent level of sampling within the same sections from the same animals. Therefore, the results reveal no differences in accuracy and precision across the three methods for volume (size) estimation, with the semiVCS and autoVCS approaches increasing efficiency for the procedure by 2 and 8 times, respectively, compared to manual data collection.

Table 3. Throughput (sections/time,t) for estimation of $V_{V}$ using autoVCS.

\begin{tabular}{lccc}
\hline Animal \# & total \# sections & total time $(\mathrm{min})$ & throughput \\
\hline 1 & 8 & 27 & 0.30 \\
2 & 8 & 14 & 0.60 \\
3 & 7 & 12 & 0.60 \\
\hline
\end{tabular}

Table 4. Comparison of precision (CE), data collection time ( $t$ ) and efficiency [CE/time) $\left.\bullet 10^{-4}\right]$ for manual point counting, semiVCS, and autoVCS for $n=3$ mice.

\begin{tabular}{llccccc}
\hline Animal\# & method & \#sections & \#frames & CE & $\mathrm{t}(\mathrm{min})$ & efficiency \\
\hline 1 & manual & 8 & 116 & 0.12 & 141 & 0.85 \\
2 & manual & 8 & 163 & 0.09 & 154 & 0.58 \\
3 & manual & 7 & 142 & 0.10 & 145 & 0.69 \\
1 & semiVCS & 8 & 123 & 0.10 & 90 & 11 \\
2 & semiVCS & 8 & 164 & 0.12 & 81 & 15 \\
3 & semiVCS & 7 & 140 & 0.12 & 75 & 16 \\
1 & autoVCS & 8 & 122 & 0.10 & 27 & 37 \\
2 & autoVCS & 8 & 169 & 0.09 & 14 & 64 \\
3 & autoVCS & 7 & 137 & 0.10 & 12 & 83 \\
\hline
\end{tabular}

Table 5. Mean BrDU volume fraction $\left(V_{V}\right)$, within-animal precision (CE) and efficiency [(mean CE/mean time) $\left(\bullet 10^{-4}\right)$ ] for manual, semiVCS, and autoVCS $(n=3$ animals $)$.

\begin{tabular}{lcccc}
\hline Method & mean V/V(SEM) & mean CE & mean t $(\min )$ & mean efficiency \\
\hline Manual & $0.0446(0.0034)$ & 0.10 & 146 & 6.8 \\
SemiVCS & $0.0460(0.0030)$ & 0.11 & 82 & $13^{* *}$ \\
AutoVCS & $0.0431(0.0028)$ & 0.10 & 18 & $55^{*}$ \\
\hline
\end{tabular}

*sig. diff., $\mathrm{p}<0.01 ; * *$ sig.diff., $\mathrm{p}<0.002$ 


\section{DISCUSSION}

In practical terms, the VCS approach allows design-based stereological analyses of volume fraction, reference volume, and total volume of BrDU-stained nuclei for $\mathrm{n}=8$ cases in the same time required to analyze a single case $(n=1)$ using the same point counting approach assisted by a state-of-the-art computerized stereology system. The logic flow allows users to identify features of interest and then train the software to correctly identify feature hits that intersect the pixel mask for the current probe. Color gradients smooth the feature detection process and provide a sharp border between targeted pixels and background. Each sample pixel is compared against the target color to see if it matches. The maximum allowed distance is defined by the sampling standard deviation times the user specified allowed number of standard deviations. The user controls the sensitivity of the color matching by selecting the scaling factor that is multiplied by the calculated color standard deviation. The product of the average gradient and the user-provided gradient factor (a global setting) represents the threshhold value for determining if a cell is on an edge or not. The gradient at each location based on the pixel distances across the boundary, and the Sobel operator is calculated. Finally, the Sobel filter is applied to the combined color distance information, rather than each channel separately. Feature size is quantified by point counting, which is more accurate and efficient than pixel counting (Gundersen et al., 1981). Finally, performance testing of the VCS approach confirmed the specificity for a particular population of high contrast targets: Training to recognize a population of high contrast dark blue images did not mistakenly identify high-contrast red features in the same sampling frame. Though the method is arguably simple, as far as we are aware VCS represents the most sophisticated approach to date to combine image analysis with state- of-the-art systematic-random sampling and parameter analysis.

A second important finding is that the VCS program provides an increase in efficiency for features of interest with middle levels of contrast. If VCS does not achieve $100 \%$ agreement with manual selections by the user, as required for fully automatic VCS, then the user has two options: continue under SemiAutomatic (user-assisted) mode; or, end the session and attempt to improve contrast using histological staining methods. In Semi-Automatic mode the software waits for the user to visually approve, and if necessary edit, the VCS-selected probe-feature intersections before proceeding to the next sampling frame.
In practice, accuracy of VCS selections depends on the $\mathrm{S}: \mathrm{N}$ ratio for the particular feature, which varies according to biological and methodological factors that control staining intensity. In these cases the program selects a subset of probe-feature intersections subject to editing for accuracy by the user. Testing confirmed that the majority of initial selections are correct for features with middle levels of contrast, requiring the user to make a few additions i.e., 1-5 clicks; only rarely did the program make incorrect selections that required removal of clicks. Therefore, in semi-automatic mode VCS selected the majority of correct probe-feature intersections, thereby reducing time spent selecting probe-feature intersections within each sampling frame. For middle levels of contrast the Semi-automatic mode increases the efficiency of data collection to a significant extent over manual data collection, albeit less than that for fully automatic VCS. Since the majority of tissuebased stereological analysis focuses on features that can be stained for at least middle levels of contrast, semi-automatic mode for VCS is expected to increase efficiency on broad range of biomedical research projects.

The advent of VCS shifts the rate-limiting step from labor-intensive user interactions with targeted images to the generation of high contrast images that allow the program to recognize the targeted feature. To minimize time required for program training, VCS includes carryover of auto-detection for features of interest on the first few sections of the first cases to subsequent sections/slides/cases within the same study, thus eliminating the need for retraining. Optimal staining for VCS includes minimal variation in staining across the population of targeted objects, which increases the efficiency of training. For features stained to reveal at least mid levels of contrast, VCS shows remarkable sensitivity to fine distinctions in contrast between target features and background. Failure to achieve maximal contrast would favor the less efficient semi-automatic VCS mode. A major factor in the overall efficiency of the VCS approach is the ability to analyze as many sections as possible on the same slide. For most reference spaces obtained from small experimental animals (e.g., mouse and rats) and many samples of human tissues, multiple sections can be mounted on a single slide. In these cases the automatic VCS feature supports automatic analysis of all sections for one animal at a single setting, without interruption to change slides, thereby reducing the time to analyze each animal in $30 \mathrm{~min}$ or less. 
The VCS approach can be readily applied to a wide variety of biomedical research projects focused on the quantitative analysis of high contrast features of biological interest. As shown in the present study, VCS could be applied to the auto-detection and autoanalysis of BrDU-positive cells following a variety of potential interventions designed to affect neurogenesis (Lee et al., 2003). The VCS approach can be expected to accelerate the collection of design-based stereology data include predictive studies of cancers where changes in volume-weighted mean nuclear volume of BrDUstained cells provides a reliable indicator of probability to metastasize (Sorensen, 1989; Masson et al., 1992; Madsen and Schroder, 1994; Ladekarl, 1998).

Though the time and effort saved by VCS could be used to sample a greater number of frames or sections from each animal, that strategy would quickly reach a point of diminishing returns (Gundersen and Jensen, 1987; Gundersen et al., 1999; West, 1999; Mouton, 2002). Because the estimation process is based on probability theory, optimal within-animal sampling to reveal significant group differences occurs after analysis of about 100 to 200 systematicrandom spaced locations on 6-10 sections through the reference space on each case. Negligible gains in precision occur beyond this level of sampling effort, while there is a drastic reduction in efficiency for the process, analogous to the cumulative probability of hitting heads or tails after flipping a coin over 200 times (50:50). As expected, sampling small frames in a large number of locations within a defined reference space achieved comparable accuracy and precision, but with higher efficiency, than large amount of data (i.e., whole images) at fewer locations. Furthermore, the additional time required to analyze the whole image at each X-Y location reduced the efficiency of procedure, without added benefits in terms of accuracy or precision to the volume estimate. Once the minimal level of sampling within each case is achieved, the major contribution to the observed variation arises from natural inter-animal variation, i.e., case-to-case differences. Thus, the most efficient strategy to reduce observed variation in the results is to repeat the minimal analysis in more cases from each group, also known by the adage, "Do More, Less Well," coined by the Swiss stereologist Ewald Weibel (see Gundersen and Osterby, 1981; Gundersen and Jensen, 1987).

In conclusion, these findings provide the first demonstration of verified computerized stereoanalysis for automatic quantification of size parameters for high contrast features of biological interest. This innovative approach takes advantage of the high efficiency of autodetection approaches associated with image analysis and avoids the introduction of stereo-logical bias that characterized previous attempts to automate quantitative morphometry using assumption- and model-based stereology methods. Future studies include extrapolation of the VCS approach to virtual probes in 3-D in order to provide automatic analysis of the full spectrum of first-and second-order stereo-logical parameters available for studies of biological tissue.

\section{ACKNOWLEDGMENTS}

This work was funded by U.S. Public Health Service NIH Small Business Innovative Research grants to the SRC and SPA. The authors would like to thank Dr. Mark P. Mattson for providing tissue used in the verification studies.

\section{REFERENCES}

Cavalieri B (1635). Geometria Indivisilibus Continuorum. Typis Clementis Ferronij. Bononiae. Reprinted 1966 As Geometria Degli Indivisibili. Unione TipograficoEditrice Torinese, Torino.

Delesse MA (1847). Procédé mécanique pour déterminer la composition des roche. Compte-Rendus D' Academie Des Sciences, Paris 25, 544-5.

Glagolev AA (1933) On the geometrical methods of quantitative mineralogic analysis of rocks. Trans Institute Econ Min 59:1.

Gundersen HJG, Østerby R (1981). Optimizing sampling efficiency of stereological studies in biology: Or "Do more less well." J Microsc 121:65-73.

Gundersen HJG, Boysen M, Reith A (1981). Comparison of semiautomatic digitizer-table and simple point counting performance in morphometry. Virchows Arch 37:3-45.

Gundersen HJG, Bendtsen TF, Korbo L, Marcussen N, Møller A, Nielsen K et al. (1988a). Some new, simple, and efficient stereological methods and their use in pathological research and diagnosis. APMIS 96:379-94.

Gundersen HJG, Bagger P, Bendtsen TF, Evans SM, Korbo L, Marcussen N et al. (1988b). The new stereological tools: disector, fractionator, nucleator and point sampled intercepts and their use in pathological research and diagnosis. APMIS 96:857-81.

Gundersen HJG, Jensen EB (1987). The efficiency of systematic sampling in stereology and its prediction. $\mathrm{J}$ Microsc 147:229-63.

Gundersen HJG, Jensen EV, Kieu K, Nielsen J (1999). The efficiency of systematic sampling in stereology revisited. J Microsc 193:199-211.

Hilliard JE, Lawson LR (2003). Stereology and Stochastic Geometry, Vol. 28. Kluwer Academic Press. 
Ladekarl M (1998). Objective malignancy grading: a review emphasizing unbiased stereology applied to breast tumors. APMIS 106:1-34.

Lee J, Auyeung WW, Mattson MP (2003). Interactive effects of excitotoxic injury and dietary restriction on microgliosis and neurogenesis in the hippocampus of adult mice. Neuromolecular Med 4:179-96.

Madsen C, Schroder HD (1994). Stereological analysis of nuclear volume in recurrent meningiomas. J Neuropathol Exp Neurol 53:272-5.

Masson E, Herlin P, Bloyet D, Duigou F, Mandard AM (1992). Development of an automatic program for the stereological estimation of the volume-weighted mean volume of cell nuclei. Path Res Pract 188:438-43.

Mouton PR, Martin LJ, Calhoun ME, Dal Forno G, Price DL (1998). Cognitive decline strongly correlates with cortical atrophy in Alzheimer's dementia. Neurobiology Aging 19:371-7.
Mouton PR (2002). Principles and practices of unbiased stereology: An introduction for biomedical scientists. Baltimore: The Johns Hopkins University Press.

Perry TA, Weerasuriya A, Mouton PR, Holloway H, Greig NH (2004). Pyridoxine-induced toxicity in rats: a stereological quantification of the sensory neuropathy. Experi Neurol 190:133-44.

Saper CB (1996). Any way you cut it: A new journal policy for the use of unbiased counting methods. J Comp Neurol 354:5.

Sorensen FB (1989). Stereological estimation of nuclear volume in benign malanocytic lesions and cutaneous malignant melanomas. Am J Dermatopathology 11: 517-27.

West MJ (1999). Stereological methods for estimating the total number of neurons an synases: issues of precision and bias. Trends Neurosci 22:51-61. 